\title{
The X-ray and EUV Turnoff of GQ Mus and V1974 Cyg
}

\section{S. STARRFIELD, ${ }^{1}$ J. KRAUTTER, ${ }^{2}$ S. N. SHORE ${ }^{3}$ I. IDAN,${ }^{4}$ G. SHAVIV, ${ }^{4}$ AND G. SONNEBORN ${ }^{5}$}

\author{
${ }^{1}$ Dept. of Physics \& Astronomy, Arizona State University, Tempe, AZ 85287, USA \\ ${ }^{2}$ Landessternwarte, Königstuhl, D69117, Heidelberg, Germany \\ ${ }^{3}$ Dept. of Physics and Astronomy, Indiana University at South Bend, \\ 1700 Mishawaka Ave, South Bend, IN 46634, USA \\ ${ }^{4}$ Dept. of Physics, The Technion, 32000 Haifa, Israel \\ ${ }^{5}$ Lab. for Astr. and Solar Phys., Code 681, NASA Goddard Space Flight Center, \\ Greenbelt, MD 20771, USA
}

\begin{abstract}
Both GQ Mus and V1974 Cyg were observed to turnoff in X-rays by ROSAT. The turnoff of V1974 Cyg was also observed with EUVE. GQ Mus was observed near the beginning of its outburst with EXOSAT and then 7 years later by ROSAT in the all-sky survey. Later ROSAT PSPC observations showed that its X-ray intensity was slowly declining with time and it was not detected in the last pointing that occurred in August 1993. We observed GQ Mus with IUE over the entire active phase of its outburst and found a change in the slope of the UV continuum around the time that the X-rays turned off. V1974 Cyg was observed by ROSAT throughout its entire active phase in X-rays which lasted about 18 months. V1974 Cyg was detected in the $E U V E$ all-sky survey, but not in pointed observations that occurred in August 1993 (and June and November 1994). We use the measured times of the active phases to determine important properties of these two novae. For example, for V1974 Cyg we predict that more than $10^{-5} M_{\odot}$ of helium rich material was left on the white dwarf when it returned to quiescence. For GQ Mus, $\sim 10^{-4} M_{\odot}$ was left on the white dwarf. These results imply that a significant amount of the helium seen in nova ejecta was produced in outbursts prior to the one that was just observed. They also imply that the mechanism which mixes core material into the ejecta must be efficient.
\end{abstract}

\section{Introduction}

It is now commonly accepted that the cause of the classical nova outburst is a thermonuclear runaway (hereafter, TNR) in the accreted hydrogen-rich envelope on the white dwarf (hereafter, WD) component of a close binary system. The secondary star in the system fills its Roche lobe and loses mass through the inner Lagrangian point into the lobe surrounding the WD. The material enters the Roche lobe of the WD with the angular momentum of the secondary and, therefore, creates an accretion disk before falling onto the WD. Hydrodynamic studies have shown that the consequence of this accretion is a growing layer of hydrogen-rich gas on the WD. When both the WD luminosity and the rate of mass accretion onto the WD are sufficiently low, so that the deepest layers of the accreted material become electron degenerate, a TNR occurs in the accreted layers. The TNR heats the accreted material $\left(\sim 10^{-4} M_{\odot}\right.$ to $\sim 10^{-6} M_{\odot}$ depending on WD mass) until an explosion occurs. This leads to the ejection of a fraction of the envelope (Starrfield 1989, and references therein). 


\section{The Constant Bolometric Luminosity Phase}

Hydrodynamic simulations have predicted, and X-ray, EUV, and IR observations have confirmed, that there is a phase of constant bolometric luminosity and high $T_{\text {eff }}$ following the initial outburst (Starrfield et al. 1972; Shore et al. 1993, 1994). The cause is that only a fraction of the accreted envelope is ejected during the initial phase of the explosion. The remaining mass (anywhere from $10 \%$ to $90 \%$ ) ultimately relaxes to quasistatic equilibrium, with a radius in the range from $\sim 10^{10}$ to $\sim 10^{11} \mathrm{~cm}$ (Truran 1982; Starrfield 1989). Because the shell source is still burning in the envelope, the luminosity of the rekindled WD stabilizes at the core-mass luminosity (Paczynski 1971; Iben 1982), which is close to $L_{\mathrm{Edd}}$ for the most massive WDs. Such a high luminosity can drive mass loss in a wind and cause the radius of the pseudo-photosphere, which occurs at some point in the expanding material, to decrease (MacDonald, Fujimoto, \& Truran 1986). Since the pseudo-photosphere shrinks in radius at constant luminosity, the $T_{\text {eff }}$ of the WD increases to values exceeding $10^{5} \mathrm{~K}$ and produces a phase of EUV or soft X-ray emission as was observed for both GQ Mus and V1974 Cyg (Ögelman et al. 1993; Krautter et al. 1995; Shanley et al. 1995; Stringfellow and Bowyer 1995). Once nuclear burning ends, this material collapses back onto the WD and cools (Starrfield et al. 1991).

One important question about the nova explosion is the length of the active phase how long does it take for nuclear burning to end and the nova to return to quiescence? Over the past three years, ROSAT has observed both the turn-on and turn-off of V1974 Cyg (Krautter et al. 1995) and the turn-off of GQ Mus (Shanley et al. 1995). We now, therefore, have measured the length of the active phase for two novae (V1974 Cyg: 18 months; GQ Mus: 9 years) plus, a measure of both the cooling time and the rate of cooling after maximum (Idan et al. 1995; Shanley et al. 1995).

\section{The X-ray and UV Evolution of GQ Mus}

GQ Mus 1983 was observed in the optical and UV near its maximum optical brightness (Krautter et al. 1984). It was also detected by EXOSAT (Ögelman, Krautter, and Beuermann 1987). During the 1980's it's optical spectrum indicated that there was a hot, photoionizing source in the system (Krautter and Williams 1989; Péquignot et al. 1993). While it was detected in 1990 during the ROSAT All Sky Survey(Ögelman et al. 1993), ROSAT PSPC observations showed that it was turning off in X-rays (Shanley et al. 1995). This was corroborated by optical observations which indicated that the nebular ionization was dropping (Hamuy et al. 1994).

GQ Mus was observed by IUE throughout its outburst. Although it was initially bright enough to obtain both SWP(1200 $\AA$ to $2000 \AA)$ and LWP (2000 $\AA$ to $3200 \AA$ ) spectra, in the past few years we have only obtained long exposure SWP observations (Idan et al. 1995). Because the nova was faint, it was necessary to correct for the residual background and camera artifacts that dominate the weakest spectra and we have developed techniques to treat these problems (Idan et al. 1995; see also Crenshaw et al. 1990). Each spectrum was then dereddened to $E(B-V)=0.45$ (Krautter et al. 1984) using the standard Galactic extinction law (Savage \& Mathis 1979). We performed pseudo-filter photometry on our spectra using a uniform weighting with $50 \AA$ bandpasses.

We chose three wavelength intervals which were found to be relatively free of emission lines. The results are shown in Figure 1, which displays the binned flux at $1375 \AA$ divided by the binned flux at $1575 \AA$ versus the binned flux at $1575 \AA$ divided by the binned flux at $1825 \AA$. Hereafter, we refer to the plotted points as the UV colors. The data for GQ Mus are marked by asterisks. We have also plotted the UV colors for hot WDs such as 


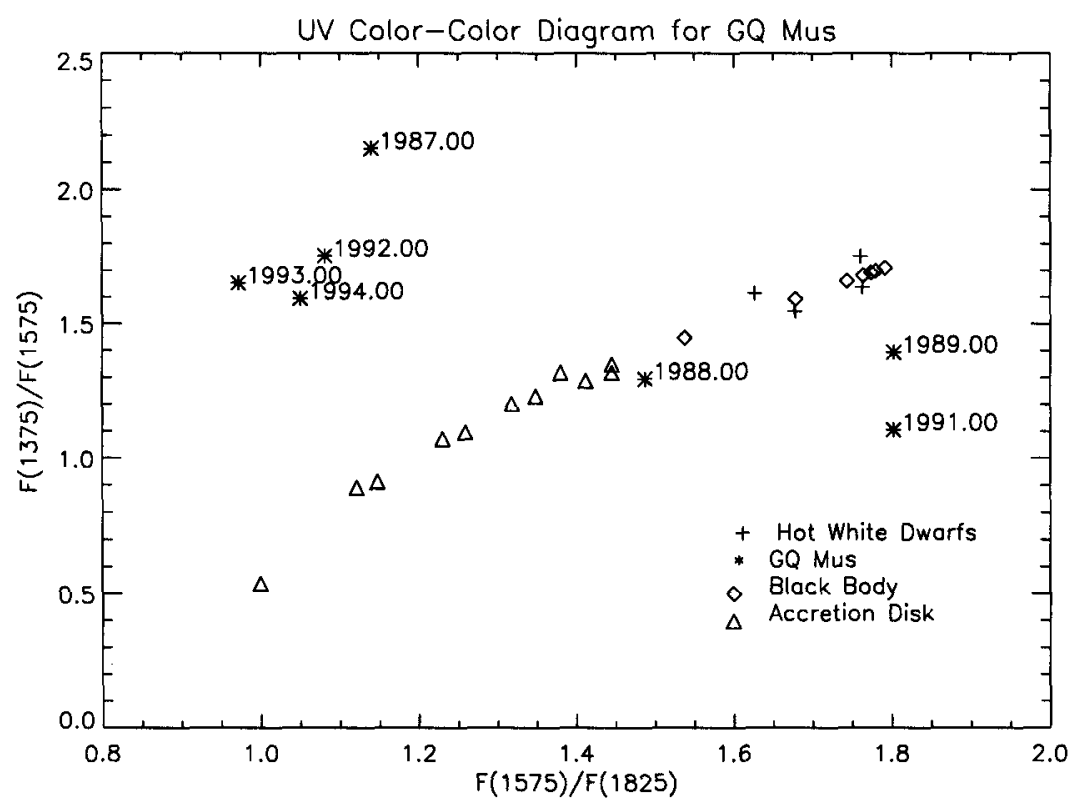

FIGURE 1. This figure displays the UV colors for GQ Mus from 1987 to 1994 . The data for GQ Mus are marked by asterisks. We have also plotted the UV colors for hot white dwarfs such as PG1159 and H1504 (+), black-bodies ranging in temperature from $5 \times 10^{4}$ to $10^{6} \mathrm{~K}(\diamond)$, and theoretical predictions for the colors of accretion disks $(\Delta)$. The errors are about $10 \%$.

PG1159 and H1504 (+), black-bodies ranging in temperature from $5 \times 10^{4}$ to $10^{6} \mathrm{~K}(\mathrm{\circ})$, and theoretical predictions for the colors of accretion disks $(\Delta)$ (Idan et al. 1995). The errors on each point for GQ Mus are probably $\sim 10 \%$. We do not plot data from before 1987 because of the presence of strong emission lines which affected the determination of the continuum.

It seems clear that the colors of GQ Mus are well separated from those of either hot WDs, black-bodies, or accretion disks. Note, however, that while the point for 1988 does fall close to those for accretion disks (for the highest mass accretion rates), that may only be a coincidence since it is evolving to the right in the diagram. We also mention that the UV colors of other novae fall in the lower-left part of this diagram and nowhere near the position of GQ Mus at any time (Idan et al. 1995). It is also interesting that the colors of this nova do not fall close to those of single WD's with $T_{\text {eff }} \sim 150,000 \mathrm{~K}$. We would have expected the continuum for the radiating WD in the GQ Mus system to have approximately the same colors as these stars if the accretion disk has not reformed.

The change in the colors from 1987 to the years 1988 through 1991 is real and indicates that the WD was increasing in temperature. This is confirmed by comparison with contemporaneous optical observations of GQ Mus which showed that the strength of $[\mathrm{Fe} \mathrm{X}] 6374 \AA$ was increasing over this time period and in 1988 its strength exceeded $\mathrm{H} \alpha$ (Krautter \& Williams 1989; Péquignot et al. 1993). During this interval its UV colors fell neither in the region where we predict that novae with accretion disks should lie nor did optical spectra show the emission lines and continuum characteristic of an accretion disk. We suggest that none was present in the system. 
The UV color-color diagram also shows that between 1991 and 1992 the nova rapidly evolved to a region of lower temperature. It was over this same interval that pointed ROSAT observations (PSPC) showed that the X-rays were turning off and it was not detected by the PSPC in late 1993 (Shanley et al. 1995). We have, therefore, determined from both UV and X-ray observations that the end of the active period of GQ Mus was in 1992 which is about 9 years after the explosion was first seen in the optical.

\section{The Importance of Measuring the Turnoff Time}

In this section we use the fact that we have obtained, for the first time, the length of the active phase for two novae. We assume that the soft X-ray emission is the result of ongoing nuclear burning in the remnant envelope on the WD which is responsible for the constant bolometric luminosity phase of the outburst (Starrfield et al. 1972, 1974).

We also need the mass of the white dwarf but this has been done neither for GQ Mus nor for V1974 Cyg from radial velocity studies. We can, however, use the observed length of the active phase to estimate the mass of the white dwarf. We do this following the discussion in Starrfield et al. (1991). We can estimate the envelope mass necessary to initiate a TNR and both calculations and analytic estimates show that that mass is a function of white dwarf mass (Starrfield 1989; Starrfield et al. 1991). We can then use the luminosity determined from the core-mass luminosity relationship (Paczynski 1971; Iben 1982 ) in combination with the theory of radiation pressure driven mass loss to calculate the mass loss rate as a function of luminosity and, hence, as a function of WD mass. Combining the two relations, allows us to obtain the length of the active phase as a function of white dwarf mass.

Using the measured intervals of the active phases for these two novae implies that the mass of the WD in GQ Mus is $\sim 1.2 M_{\odot}$ and the mass of the WD in V1974 Cyg is $\sim 1.3 M_{\odot}$. The concomitant luminosities are $\sim 5 \times 10^{4} L_{\odot}$ for V1974 Cyg and $\sim 4 \times 10^{4} L_{\odot}$ for GQ Mus. The value for V1974 Cyg is in reasonable agreement with the value determined by Shore et al. $(1993,1994)$. The value determined by Hassell et al. (1990) for GQ Mus was $\sim 2.5 \times 10^{4} L_{\odot}$ which is close enough to our estimate for the purposes of this paper. For the rest of this section, however, we shall assume a $1.25 M_{\odot}$ WD and a luminosity of $4.5 \times 10^{4} L_{\odot}$ (Iben 1982).

The analyses of the X-ray emission from these novae imply effective temperatures for the WD $\sim 3 \times 10^{5} \mathrm{~K}$. Because these values depend on black-body fits to the observed X-ray PSPC energy distributions, they are not well determined. Nevertheless, a hot, luminous $1.25 M_{\odot}$ WD radiating at the core-mass luminosity with an effective temperature of $3 \times 10^{5} \mathrm{~K}$ has a radius of $5.6 \times 10^{9} \mathrm{~cm}$. This value is 15 times larger than the equilibrium radius of a $1.25 M_{\odot}$ WD $\left(3.8 \times 10^{8} \mathrm{~cm}\right)$. Therefore, at the beginning of the $\mathrm{X}$-ray decline phase, the configuration of the remnant was that of a WD with a hot, extended envelope.

We now assume that the final decline is caused by the WD consuming all available hydrogen and the energy radiated during the decline comes only from gravitational contraction of the hot, extended, helium-rich layers. We can then use the Kelvin-Helmholtz time scale (Cox \& Giuli 1967) to obtain the envelope mass required to produce the observed energy (Krautter et al. 1995). We use a time for the collapse of the envelope of 6 months for V1974 Cyg and other parameters for a $1.25 M_{\odot}$ WD to arrive at a mass for the helium layer of $\sim 10^{-5} M_{\odot}$. This is an upper limit to the remnant envelope mass. Assuming a lower mass for the WD in GQ Mus and a cooling time scale of $\sim 3$ years (Shanley et al. 1995), implies that it ended its outburst with more than $10^{-4} M_{\odot}$ of helium on the surface. These values are significantly larger than the amount of mass 
thought to be left on the WD after the outburst (Starrfield 1989). In addition, the value for V1974 Cyg is a large fraction of the ejected mass $\left(>10^{-4} M_{\odot}\right.$ : Shore et al. 1993).

The fact that we observed the entire nuclear burning time for their outbursts allows us to determine additional parameters. We again assume constant bolometric luminosity phases of 18 months and 9 years. Therefore, their total radiated luminosities were $9 \times 10^{45} \mathrm{ergs}$ (V1974 Cyg) and $\sim 5 \times 10^{46} \mathrm{ergs}$ (GQ Mus). V1974 Cyg ejected $\sim 10^{-4} M_{\odot}$ at a speed of $\sim 2 \times 10^{8} \mathrm{~cm} \mathrm{~s}^{-1}$ (Shore et al. 1993) for a total kinetic energy of $\sim 4 \times 10^{45}$ ergs. GQ Mus ejected $\sim 10^{-4} M_{\odot}$ at a speed of $\sim 10^{8} \mathrm{~cm} \mathrm{~s}^{-1}$ (Krautter et al. 1984; Hassell et al. 1990) for a total kinetic energy of $\sim 10^{45}$ ergs. These results imply that $\sim 10^{-6} M_{\odot}$ of hydrogen was burnt to helium during their outbursts. This is less than $1 \%$ of the mass of the ejecta and suggests that the observed enrichment of helium in nova ejecta (Hassell et al. 1990; Péquignot et al. 1993; Andreä et al. 1994; Austin et al. 1995) does not come from nuclear burning but from mixing with material that was already present on the surface of the WD.

\section{Discussion}

We have assumed that the X-ray decline marked the end of the nuclear burning phase when all the hydrogen had been fused to helium. One of the consequences of the outburst on both these novae, therefore, must be a massive layer of helium on the surface of the WD. Once accretion onto the WD is again initiated, the infalling material must penetrate the helium-rich layer in order to reach to the $\mathrm{CO}$ or $\mathrm{ONeMg}$ core material which is seen in nova ejecta. This helium, however, must also be ejected because the observations also show large enrichments of helium in nova ejecta. This claim is supported by the helium enrichment found by Austin et al. (1995) for V1974 Cyg (He/H $\sim 5$ times solar). This result also implies that efficient mixing must occur between core and accreted material prior to the TNR. Therefore, the large amounts of helium observed in nova outbursts is not produced by the current outburst but in previous outbursts. In addition, if the WD is as massive as we have found in our calculations, then the accretion time scale is relatively short and the mixing mechanism must be significantly more efficient than diffusion (Prialnik and Kovetz 1984).

In summary, we have used the observed turn-off times of V1974 Cyg and GQ Mus to determine important properties of the nova outburst.

\section{REFERENCES}

ANDreä, J. , Drechsel, H. , \& Starffeld, S. 1994, A\&A, 291, 869

Austin, S. J., Wagner, R. M., Starrfield, S., Shore, S. N., Sonneborn, G., \& Bertram, R. 1995, ApJ, in preparation

Crenshaw, D. M., Norman, D. J., \& Bruegman, O. W. 1990, PASP, 102, 463

Hamuy, M., Phillips, M., \& WILliams, R. E. 1994, IAUC \#5953

Hassell, B. J. M. et al., 1990, in Physics of Classical Nova, ed. A. Cassatella \& R. Viotti, Berlin: Springer-Verlag, NY, 202

IBEN, I. JR. 1982, ApJ, 259, 244

Idan, I., Starrfield, S., Shore, S., Krautter, J., Sonneborn, G., \& Shaviv, G. 1995, ApJ, in preparation

Krautter, J., et al., 1984, A\&A, 137, 307

Krautter, J., Ögelman, H., Starrfield, S., WichmanN, R. \& Pfefferman, R., 1995, ApJ, in press

Krautter, J. \& Whliams, R. E. 1989, ApJ, 341, 968 
MacDonald, J., Fujimoto, M. Y., \& Truran, J. W. 1985, ApJ, 294, 263

Ögelman, H., Krautter, J., \& Beuermann, K. 1987, A\&A, 177, 110

Ögelman, H., Orio, M., Krautter, J., \& Starrfield, S. 1993, Nature, 361, 331

PaczYNSKI, B. 1971, Acta Astron., 21, 271

PÉquignot, D., Petitjean, P., Boisson, C., \& Krautter, J. 1993, A\&A, 271, 219

PrialNIK, D. \& Kovetz, A. 1984, ApJ, 281, 367

SaVAGE, B., \& Mathis, J. 1979, ARAA, 17, 73

Shanley, L., Ögelman, H., Gallagher, J. S., Orio, M., \& Krautter, J. 1994, ApJl, 438, L95

Shore, S. N., Sonneborn, G., Starrfield, S., Gonzelez-Riestra, R., \& Ake, T. B. 1993, AJ, 106, 2408

Shore, S. N., Sonneborn, G., Starrfield, S., Gonzelez-Riestra, R., \& Polidan, R. S. 1994, ApJ, 421, 344

Starffield, S. 1989, in Classical Novae, ed. M. Bode and A. N. Evans, Wiley Press, New York, 39

StARRFIELD, S. 1992, in Variability in Stars and Galaxies: Reviews in Modern Astronomy, 5, ed. G. Klare, Heidelberg: Springer-Verlag, 73

Starrfield, S., Sparks, W. M., \& Truran, J. W. 1974, ApJS, 28, 247

Starrfield, S., Truran, J. W., Sparks, W. M., \& Kutter, G. S. 1972, ApJ, 176, 169

Starrfield, S., Truran, J., Sparks, W. M., \& Krautter, J. 1991, in Extreme Ultraviolet Astronomy, ed. R. Malina, \& S. Bowyer, 168

Stringfellow, G., \& Bowyer, C. S. 1995, in Proceedings EUVE IAU Colloquium, ed. S. Bowyer, Berkeley, CA, these proceedings

Truran, J. W. 1982, in Essays in Nuclear Physics, ed. C. A. Barnes, D. D. Clayton, and D. N. Schramm, Cambridge, University Press, 467 